\title{
Eficiência Produtiva das Fazendas de Carcinicultura no Estado do Ceará ${ }^{1}$
}

\author{
Jair Andrade Araujo ${ }^{2}$, Alana Kedylla Monteiro Norões ${ }^{3}$, \\ Jonathas Viana Monteiro ${ }^{4}$, Rogério César Pereira de Araújo ${ }^{5}$ e \\ Felipe Pinto da Silva ${ }^{6}$
}

Resumo: A eficiência produtiva da carcinicultura tem sido apontada na literatura como um fator importante para a consolidação desta atividade no Nordeste do Brasil. Para auxiliar a tomada de decisão na fazenda e a formulação de políticas para o setor, esta pesquisa investigou o grau e os fatores determinantes da eficiência técnica na produção de camarão no estado do Ceará. Com base nos dados do Censo da Carcinicultura, de 2011, coletados pela Associação Brasileira de Criadores de Camarão (ABCC), 100 fazendas foram caracterizadas por um conjunto de variáveis explicativas que descrevem os insumos empregados na produção e a forma de gerenciamento das fazendas. Para analisar os dados, foram estimados modelos de fronteira de eficiência de produção estocástica. Os resultados mostraram que quase a totalidade das fazendas $(99 \%)$ pode ser considerada ineficiente do ponto de vista técnico. $\mathrm{O}$ fator que determinou a ineficiência das fazendas da amostra foi a densidade média de estocagem, pois as outras variáveis testadas como causadoras de ineficiência (que foram: potência de aeradores por área, recebimento de assistência técnica e uso de bandejas de alimentação) não foram significantes a um nível de significância de $5 \%$. Com isso, conclui-se que estas variáveis não geram ineficiência no modelo.

Palavras-chaves: camarão, eficiência técnica, fronteira estocástica.

Abstract: The productive efficiency of shrimp farming has been pointed out in the literature as an important factor for the consolidation of this activity in Northeast Brazil. In order to assist decision making on the farm and the formulation of policies for the sector, this research investigated the degree and determinants of technical efficiency in shrimp production in Ceará State. Based on data from the Carcinculture Census, collected by the Brazilian Association of Shrimp Breeders (ABCC, 2011), 100 farms were characterized by a set of explanatory variables that describe the inputs used in the production and the way of managing the farms. To analyze data, we estimated frontier models

1. Data de submissão: 21 de setembro de 2016. Data de aceite: 21 de julho de 2017.

2. Universidade Federal do Ceará. Fortaleza, Ceará. Brasil. E-mail: jaraujoce@gmail.com

3. Universidade Federal do Ceará. Fortaleza, Ceará. Brasil. E-mail: alanadylla@hotmail.com

4. Universidade Federal do Ceará. Fortaleza, Ceará. Brasil. E-mail: jonathas0801@ hotmail.com

5. Universidade Federal do Ceará. Fortaleza, Ceará. Brasil. E-mail: rcpa@ufc.br

6. Universidade Federal do Ceará. Fortaleza, Ceará. Brasil. E-mail: dasilva.felipe0@gmail.com 
of stochastic production efficiency. The results showed that almost all farms (99\%) can be considered technically inefficient. The factor that determined the inefficiency of the sample farms was the average storage density, since other variables tested as inefficiency (which were: power of aerators per area, receipt of technical assistance and use of feed trays) were not significant at a significance level of 5\%. As a result, these variables do not generate inefficiency in the model.

Key-words: shrimp, technical efficiency, stochastic frontier.

Classificação JEL: O47; O54; O57.

DOI: http://dx.doi.org/10.1590/1234-56781806-94790560103

\section{Introdução}

A carcinicultura é um dos ramos da aquicultura que mais tem crescido no mundo. Em 2012, o Brasil contribuiu com $55 \%$ do total da produção mundial de camarões e com $15 \%$ do valor total de produtos aquícolas comercializados mundialmente (FAO, 2014).

No Brasil, a carcinicultura marinha, que consiste na criação de camarões, teve início na década de 1970, mas somente passou a ter investimentos privados a partir da década seguinte, com a produção de camarões peneídeos (Marsupenaeus japonicus, Litopenaeus schmitti, Farfantepenaeus subtilis, F. brasiliensis e F. paulensis). A partir dos anos 1990, a atividade experimentou crescimento significativo por meio da introdução da espécie exótica Litopenaeus vannamei, conhecida como camarão branco do Pacífico (MAGALHÃES, 2004).

Em 2011, a produção nacional foi de 65.671 toneladas, representando $80 \%$ da produção total aquícola marinha naquele ano (FAO, 2014). Segundo Araújo (2001), a espécie Litopenaeus vannamei tem produtividade elevada, podendo alcançar mais de $6.000 \mathrm{~kg} / \mathrm{ha} /$ ciclo. Em 2002, a produtividade média brasileira foi de $5.458 \mathrm{~kg} / \mathrm{ha} / \mathrm{ano}$, sendo a maior registrada no mundo naquele ano (ROCHA e RODRIGUES, 2003).

A cadeia produtiva de camarão cultivado no Brasil já é bastante consolidada e tem se expandido cada vez mais à medida que o número de fornecedores de insumos vem crescendo no país, como é o caso das indústrias de insumos (ração, medicamentos etc.), laboratórios de pós-larvas e centros de processamento.

A carcinicultura tem gerado benefícios econômicos e sociais no Brasil, principalmente na região Nordeste. Em 2011, a cadeia produtiva do camarão cultivado - constituída de laboratórios de pós-larvas, fazendas de engorda e centros de processamento - gerou $\mathrm{R} \$ 1$ bilhão (ABCC, 2013). Sabe-se que esta atividade gera 1,89 emprego direto e 1,86 emprego indireto por hectare de viveiro em produção, totalizando 3,75 empregos por hectare. Este valor de empregos supera setores primários tradicionais da Zona da Mata, como as cadeias produtivas da cana-de-açúcar e do coco, como também setores de destaque regional, como a fruticultura dos polos irrigados. A carcinicultura tem se colocado como uma alternativa econômica promissora para os municípios, que se encontram estagnados ou em declínio econômico (SAMPAIO e COUTO, 2008).

O maior número de fazendas de carcinicultura no Brasil está na região Nordeste, particularmente nos estados de Sergipe, Alagoas, Pernambuco, Paraíba, Rio Grande do Norte, Ceará e Piauí, que concentraram, em 2011, mais de 99\% (ou 69.088 t) da produção de camarão cultivado (RODRIGUES e BORBA, 2013).

Nesse mesmo ano, o estado do Ceará se destacou como o maior produtor, com total de $31.982 \mathrm{t}$ e área em operação de 6.580 hectares (ABCC, 2013). Segundo o censo realizado pela ABCC (2013), o Ceará experimentou crescimento em número de fazendas e produção, entre 2004 e 2011, de $41,24 \%$ e $61 \%$, respectivamente.

Este aumento da produção e produtividade da carcinicultura no Brasil tem sido atribuído à própria capacidade da espécie cultivada de se adaptar às condições edafoclimáticas (ABCC, 2013), à expansão da área cultivada (RODRIGUES e BORBA, 2013), ao uso de produtos biotecnológicos (JONES e DOURADO, 2003), à alta densidade de estocagem dos viveiros (FONSECA, 2005) e à melhoria da gestão da propriedade (SANTOS et al., 2015). Porém, a qualidade inferior dos insumos pode ser um fator limitante para o crescimento da 
produtividade do cultivo de camarão (OSTRENSKY e PESTANA, 2000).

Além disso, o cenário da carcinicultura no estado do Ceará teve mudança na primeira década do século XXI, principalmente pelo aumento do número de fazendas, pela crise financeira internacional e pelo embargo das importações para os EUA. Estes fatos podem ter ocasionado mudanças significativas no desempenho produtivo da carcinicultura devido ao aumento da competição por insumos entre as fazendas e à necessidade de combinar e/ou substituir insumos para alcançar a eficiência técnica (ABCC, 2013).

Desta forma, este estudo tem como objetivo avaliar a eficiência técnica da carcinicultura marinha cearense, com base nos dados do Censo Brasileiro da Carcinicultura de 2011. E, especificamente, estimar a função de produção e realizar uma comparação entre os produtores mais e menos eficientes. Espera-se, com isto, capturar as mudanças ocorridas no setor nas últimas duas décadas na produção de camarão marinho, cultivado no estado do Ceará. Para isso, foi utilizado o método de fronteira estocástica, que permite estimar o nível de eficiência técnica da unidade produtiva. No Brasil, são escassos os estudos realizados com aplicação deste método em atividades agrícolas sendo praticamente inexistentes para o setor aquícola cearense.

Além desta introdução, o artigo é composto por mais quatro seções. Na segunda seção foi apresentado o nível de produtividade do camarão e os aspectos teóricos e metodológicos da eficiência técnica e fronteira de produção. Foi feita também uma revisão de literatura sobre a aplicação da fronteira de produção estocástica na carcinicultura. Na terceira seção apresentou-se a metodologia da pesquisa com destaque para a área de estudo, natureza e fonte dos dados e o modelo econométrico da fronteira de eficiência estocástica. Na quarta seção foram apresentados e discutidos os resultados e, finalmente, na quinta seção, foram expostas as considerações finais.

\section{Referencial teórico}

\subsection{Eficiência técnica e fronteira de produção}

A análise da eficiência em uma unidade de produção pode auxiliar a tomada de decisão na propriedade visando a melhoria de seu desempenho. Um processo de produção é constatado eficiente por meio da comparação da sua situação atual com uma situação ótima que poderia ser atingida dada as combinações de insumos ou de produtos (SOUZA JÚNIOR, 2003).

Farrel (1957) foi o pioneiro em estudos sobre eficiência econômica e concentrou-se na medição da eficiência com relação à utilização dos insumos. Conceitualmente, eficiência econômica pode ser definida como o resultado máximo obtido, de acordo com os fatores empregados, ou ainda como a capacidade da empresa em utilizar os fatores de produção em proporção ótima, minimizando os custos de produção. Já a eficiência técnica é uma medida do modo como a combinação ótima dos recursos é utilizada na produção, na busca do produto máximo. Enquanto a eficiência técnica está preocupada com o aspecto físico da produção, a eficiência econômica é uma extensão da eficiência técnica, que se preocupa com aspecto monetário da produção (SOUZA JÚNIOR, 2003).

Sabe-se que nem todos os produtores são tecnicamente eficientes, ou seja, nem todos conseguem utilizar a quantidade mínima de insumos necessária para produzir a quantidade de produto desejada, dada a tecnologia disponível (RIVERA e CONSTANTIN, 2007).

Segundo Tupy e Yamagucho (1998), ainda que muitos autores considerem o crescimento da produtividade e a eficiência como sinônimos, existe um pequeno, porém crescente grupo que difere os dois conceitos. O crescimento da produtividade pode ser definido como a mudança líquida no produto devido às mudanças na eficiência e mudanças tecnológicas, em que a primeira é a variação do produto observado em relação à sua fronteira, e a segunda representa $\mathrm{o}$ deslocamento da fronteira de produção.

Conforme Conceição e Conceição (2005), a literatura recente apresenta avanços na obtenção das estimativas de eficiência, a partir da estimação de funções de produção, utilizando, principalmente, a função de fronteira de produção estocástica. A modelagem econométrica de funções de fronteira de produção fornece a produção máxima, que pode ser obtida a partir de um determinado conjunto de insumos, dada a tecnologia existente para as firmas envolvidas no processo produtivo.

As principais abordagens metodológicas utilizadas para a estimação da fronteira são, a saber: abordagem conhecida como não paramétrica; a outra é chamada de paramétrica (LOVELL e SCHMIDT, 1988). 
O método não paramétrico, como o Data Envelopment Analysis (DEA), utiliza uma sequência de programas lineares para construir uma fronteira de produção e, assim, mensurar a eficiência relativa (SILVA, CARVALHO e CAMPOS, 2007). Já a abordagem paramétrica faz uso de técnicas para estimar a fronteira e computar a sua ineficiência. Nesta pesquisa, o modelo de fronteira de produção estocástica foi o método que mais se adaptou à análise das variáveis.

A metodologia de fronteira estocástica admite desvios da fronteira e, para isso, adiciona um componente de erro para capturar influências causadas por ruído e erros de medição. Tal artifício possibilita a divisão do desvio de uma observação da parte determinística da fronteira em dois constituintes: um associado à ineficiência técnica da produção e outro relacionado aos ruídos aleatórios (REIS, RICHETTI e LIMA, 2005).

Este método de fronteira estocástica (FE) utiliza técnicas econométricas para estimar a fronteira de produção que, por sua vez, caracteriza uma transformação eficiente de insumos e produtos, além de computar a sua eficiência relativa. Para isto, define-se uma forma funcional específica para a tecnologia em questão e as hipóteses distribucionais sobre os componentes do erro, de modo a permitir a sua decomposição (REIS, RICHETTI e LIMA, 2005).

O método de FE apresenta algumas limitações referentes à má especificação da distribuição assumida para o termo da ineficiência, especialmente na utilização da distribuição half-normal (LIU, LAPORTE e FERGUNSON, 2008) e à violação ocasional das propriedades da função de produção ${ }^{7}$, principalmente a monotonicidade, diferente do que ocorre no modelo de DEA, em que essas condições são satisfeitas pela construção do problema de programação linear (REICHARD, KNOX LOVELL e THJSSEN, 2000). Outra limitação apontada por Tannuri-Pianto, Sousa e Arcoverde (2009) é a imposição de uma forma funcional; entretanto, este método possibilita a mensuração de escores de eficiência na existência de firmas com multi-insumos e multiprodutos.

Essa metodologia, porém, é mais apropriada ao setor agrícola do que as abordagens determinísticas que geram fronteiras não paramétricas, especialmente quando se trata de países como o Brasil, que

7. As principais propriedades associadas a função de produção são apresentadas detalhadamente em Coelli, Rao, O'Donnell e Battese (2008). estão em desenvolvimento, em que as informações são fortemente influenciadas por erros de medição, condições climáticas, doenças e pragas (COELLI, RAO e BATTESE, 1998). Desta forma, muitos estudos relacionados à estimação da eficiência técnica têm sido realizados com utilização da metodologia paramétrica da estimativa da função fronteira estocástica, sendo bastante utilizada nos trabalhos empíricos e, principalmente, naqueles aplicados à agropecuária (ALMEIDA, 2012; DEY et al., 2010).

\subsection{Fronteira de produção estocástica na carcinicultura}

Os estudos que têm como enfoque determinar a eficiência técnica no setor aquícola são escassos, particularmente no que diz respeito à eficiência técnica da carcinicultura, o mesmo ocorrendo no Brasil.

No mundo, destacam-se as estimativas de eficiência técnica para a carcinicultura em Bangladesh (BEGUM, HOSSAIN e PAPNAGIOTOU, 2013; RASHID e CHEN, 2002), na Malásia (ISLAM, YEW e NOH, 2014) e em Andhra Pradesh (SIVARAMAN et al., 2015).

Begum, Hossain e Papnagiotou (2013) determinaram a eficiência técnica dos carcinicultores de Bangladesh usando a função de fronteira de produção estocástica, que envolve um modelo com determinantes de ineficiência técnica. Os fatores estudados como causadores de ineficiência na produção das fazendas de camarão e seus respectivos coeficientes foram: nível de escolaridade do aquicultor $(-0,166)$; participação em associações de aquicultura $(0,561)$; qualidade da água $(0,216)$; treinamento $(-0,755)$ e idade $(-0,016)$. A pesquisa foi realizada com uma amostra de 90 fazendas de camarão de água salobra, localizadas na região de Shyamnagar Upazila, em Bangladesh. A partir dos resultados dos coeficientes, foi visto que o nível de escolaridade do aquicultor, o treinamento e a idade reduzem a ineficiência do modelo, ou seja, o aumento nessas variáveis irá gerar um ganho de eficiência para o produtor; porém, a participação em associações e a qualidade da água apresenta-se como causador de ineficiência no modelo. O nível de eficiência técnica média dos carcinicultores foi estimada em $82 \%$; portanto, fazendo o setor operar $18 \%$ abaixo da fronteira de produção.

Rashid e Chen (2002) também estudaram a eficiência técnica de três sistemas de cultivo de camarão marinho (extensivo, semi-intensivo e intensivo) de 
uma amostra de 155 fazendas localizados nas regiões sudeste e sul ocidental de Bangladesh. As variáveis utilizadas para verificação como determinantes de ineficiência foram: área da fazenda, nível de escolaridade e experiência dos funcionários. Por meio do modelo de fronteira estocástica, as médias de eficiência para os três sistemas de cultivos investigados foram 0,82, 0,85 e 0,93 , respectivamente. Foi verificado que, em todos os sistemas de cultivo, todas as variáveis testadas como determinantes de ineficiência obtiveram coeficientes negativos, com exceção da variável área da fazenda no sistema de cultivo semi-intensivo, demostrando que o aumento destas reduziria a ineficiência do modelo.

Islam, Yew e Noh (2014) analisaram a eficiência técnica da carcinicultura na península da Malásia utilizando a abordagem de fronteira de produção estocástica. Os fatores de ineficiência testados no modelo foram: área da fazenda, número de ciclos produtivos, nível educacional, idade e experiência dos funcionários. Dentre os resultados foram ressaltados os coeficientes das variáveis experiência $(-0,011)$ e educação $(-0,018)$, que foram negativos, sendo que um aumento destas variáveis reduziria a ineficiência das fazendas, ou seja, quanto maior for o nível educacional e a experiência dos funcionários menor será a ineficiência do modelo. Concluíram que, nas fazendas de camarão estudadas, existia alto grau de ineficiência técnica entre os criadores de camarão, evidenciando, assim, grande potencial para aumentar a produção de camarão por meio da melhoria da eficiência na gestão das explorações agrícolas nessa região.

Sivaraman et al. (2015) estimaram uma função de fronteira de produção estocástica para analisar a eficiência técnica das fazendas de camarão do distrito de Godavari Oriental de Andhra Pradesh. As variáveis analisadas como causadoras de ineficiência foram: participação em associações de carcinicultores, experiência, escolaridade, idade e tamanho da família dos carcinicultores. Observaram que a experiência, idade e escolaridade dos carcinicultores têm efeitos positivamente associados com a eficiência técnica. Esses resultados sugerem que os produtores com mais experiência, mais idade e melhor educação tomam decisões mais adequadas quanto ao manejo da fazenda e, assim, aumentam sua eficiência técnica. A eficiência técnica dos carcinicultores foi estimada em 93,06\%, o que significa que as fazendas operavam em 6,94\% abaixo da produção de fronteira de produção.
No Brasil, as análises de eficiência técnica da carcinicultura são em pequeno número, com destaque para os trabalhos de Sousa Júnior (2003), Silva e Sampaio (2009) e Oliveira (2008), todos realizados com dados da região Nordeste.

Sousa Júnior (2003) analisou a eficiência da produção de camarão marinho em cativeiro no estado do Ceará, em 2002, utilizando a Análise de Envoltória de Dados (DEA). Os resultados mostraram que, no ponto de vista técnico, os carcinicultores eram eficientes. Para isto foi selecionada uma gama de variáveis importantes na produção, para verificar quantos destes produtores eram eficientes, como densidade de estocagem, aeração artificial, espécie utilizada, oxigênio dissolvido etc. De uma amostra de 68 fazendas, 38 deles (ou 56\%) se mostraram tecnicamente eficientes, obtendo medidas de eficiência técnica dentro do intervalo de 0,9 a 1,0, enquanto outros 30 foram considerados tecnicamente ineficientes.

Silva e Sampaio (2009) estimaram a fronteira de produção não paramétrica e eficiência técnica para dois sistemas de produção (extensivo e semi-intensivo) conduzidos por pequenos e médios produtores de camarão do estado do Rio Grande do Norte, em 2009. A partir de uma amostra de 348 pequenos e médios produtores de camarão (com área inferior a 50 ha), obtida do censo da carcinicultura realizado pela ABCC, em 2004, observou-se que os níveis de eficiência técnica foram mais altos para os sistemas de produção extensivo e semi-intensivo e que algumas práticas de gestão estão associadas diretamente aos scores de eficiência, como o uso de aeradores, equipamentos para monitoração da água, tratamento de solo, utilização de tanques berçários etc. Entretanto, algumas variáveis de gestão mostraram-se não significativas, como a presença de bandejas para ração, que pode ser explicada pela falta de conhecimento do nível adequado do balanceamento da ração, que deve ser empregado nas bandejas. Constatou-se também que produtores localizados em estuários com menor nível de emissões de nitrogênio e fósforo e, portanto, menos poluentes na média, obtiveram maior índice de eficiência técnica.

Oliveira (2008) avaliou a eficiência do cultivo de camarão marinho, em sistema dulciaquícola numa fazenda localizada no Rio Grande do Norte, no período compreendido entre 2002 e 2005. O autor utilizou a Análise de Envoltória de Dados (DEA) para trabalhar com um banco de dados composto por 115 observações 
e considerou as seguintes variáveis para verificar a eficiência do cultivo: variáveis de produto, produção e peso médio final; variáveis de insumo, tempo de cultivo, quantidade de pós-larva, área do viveiro, quantidade de ração ofertada, marca da ração e laboratório de origem das pós-larvas. Os resultados demonstraram que 24 cultivos se mostraram eficientes e 91, ineficientes, e que a variável exógena temperatura foi relacionada com a produção e com o peso médio final dos camarões, enquanto que oxigênio, $\mathrm{pH}$ e transparência não tiveram relação significativa ( $p \geq 0,05)$.

\section{Metodologia}

\section{1. Área de estudo}

O estudo restringe-se às fazendas de carcinicultura de portes micro, pequeno, médio e grande, especializadas na engorda de camarão e localizadas no estado do Ceará. Em 2011, o Ceará tinha 325 produtores de camarão que ocupavam área de 6.580 ha de viveiros em operação, com área média de 20,2 ha/fazenda, distribuídos em 21 municípios. Naquele ano, a produção de camarão foi de $31.982 \mathrm{t}$, tendo alcançado produtividade média de 4,86 t/ha/ano.

Os municípios que se destacaram em 2011 foram Aracati, que assumiu a primeira posição em número de produtores, área cultivada e produção, seguido de Acaraú, Camocim, Jaguaruana, Beberibe e Fortim - todos com produção individual superior a $1.000 \mathrm{t}$ anuais (ABCC, 2013).

\subsection{Natureza e fonte dos dados}

Os dados utilizados nesta pesquisa, referentes à carcinicultura no estado do Ceará, são de natureza secundária, tendo sido extraídos do Censo Setorial do Camarão, realizado pela Associação Brasileira de Criadores de Camarão (ABCC), em 2011.

Segundo a ABCC (2013), os dados foram coletados por meio de pesquisa direta, com utilização da metodologia universal de censos, que consiste da localização das unidades produtivas, georreferenciamento e aplicação de questionários para coletar informações sobre os aspectos físicos, sociais e ambientais da produção. A pesquisa direta foi feita com os atores sociais envolvidos na carcinicultura estadual e conduzida pelo setor técnico da associação, com o apoio de consultorias especializadas.

No Ceará, 325 produtores foram pesquisados e as entrevistas realizadas na própria fazenda, assegurando, assim, a confiabilidade dos dados (ABCC, 2013).

As variáveis do modelo correspondem a todo o período de 2011 e a escolha das variáveis que formam a fronteira de produção foi baseada no fato de que, para a estimação da função de produção, é necessário que a variável dependente do modelo represente a produção e que as variáveis explicativas correspondam aos insumos necessários a essa produção. Desta forma, tem-se como variável dependente a produção de camarão em toneladas, que representa a capacidade de cada fazenda em produzir camarão, levando-se em consideração que cada firma pretende sempre alcançar maior nível de produção. Por isso, essa variável é bastante utilizada para estudos relacionados à carcinicultura, como pode ser visto naqueles realizados por Sousa Júnior (2003) e Silva e Sampaio (2008). Já em relação às variáveis independentes do modelo, tem-se que: com o uso da variável explicativa, número total de funcionários contratados, visa-se medir a força do trabalho nessa atividade e verificar a importância dela para a produção das fazendas, verificando-se também se este será um dos principais insumos produtivos, pois, na maioria das fazendas de camarão, o número de funcionários contratados é bem aquém comparado à dimensão das fazendas; as variáveis número de viveiros em operação e área média dos viveiros em hectares irão representar a magnitude das fazendas, ressaltando a área que é utilizada na produção, podendo-se, assim, verificar se a terra está sendo utilizada de forma a maximizar a produção, sendo estas variáveis explicativas de grande importância e, por isso, já utilizada por outros autores, como Rashid e Chen (2002), Begum, Hossain e Papnagiotou (2013) e Islam, Yew e Noh (2014).

A Tabela 1 mostra as estatísticas descritivas das variáveis utilizadas nos modelos. 
Tabela 1. Estatística descritiva das variáveis utilizadas na estimação da fronteira de produção

\begin{tabular}{|c|c|c|c|c|c|}
\hline Variáveis & Definição & Média & Desvio padrão & Mínimo & Máximo \\
\hline \multicolumn{6}{|l|}{ Produção $\left(Y_{i}\right)$} \\
\hline ProdC & Produção de camarão (toneladas) & 200,30 & 444,48 & 4,00 & 4150,00 \\
\hline \multicolumn{6}{|c|}{ Variáveis explicativas $\left(X_{i}\right)$} \\
\hline NFunc & Número total de funcionários contratados & 16,39 & 35,19 & 1,00 & 320,00 \\
\hline Nviv & Número de viveiros em operação & 11,91 & 23,39 & 0,50 & 220,00 \\
\hline Aviv & Área média dos viveiros (ha) & 2,81 & 1,69 & 1,00 & 12,00 \\
\hline \multicolumn{6}{|c|}{ Variáveis de ineficiência $\left(Z_{i}\right)$} \\
\hline Dens & Densidade média de estocagem $\left(\mathrm{cam} / \mathrm{m}^{2}\right)$ & 33,23 & 11,73 & 10,00 & 80,00 \\
\hline PAer & Potência de aeradores por área (HP/ha) & 6,46 & 6,42 & 0,50 & 60,00 \\
\hline Atec & Recebimento de assistência técnica & - & - & - & - \\
\hline Band & Uso de bandejas de alimentação & - & - & - & - \\
\hline Total de obse & vações estudadas -100 & & & & \\
\hline
\end{tabular}

Fonte: Dados do Censo Setorial do Camarão, ABCC (2011).

A variável ProdC, produção de camarão, é a variável dependente do modelo e refere-se ao total obtido durante os ciclos produtivos em 2011. As variáveis explicativas são: número total de funcionários contratados, o qual representa a força de trabalho; número de viveiros em operação; área média dos viveiros, em hectares; potência de aeradores por área; densidade média de estocagem, que mostra como o produtor aloca as larvas em seu espaço produtivo; recebimento de assistência técnica e uso de bandejas de alimentação, que são variáveis qualitativas que representam aspectos tecnológicos da firma, que podem assumir valor de 1 quando a resposta for Sim e 0 quando a resposta for Não.

São 325 produtores; porém, nos modelos de fronteira estocástica, não se faz uso de dados faltosos. Por esta razão, para o modelo foram utilizadas 100 observações.

\subsection{Método de análise}

O método escolhido para este artigo é o de análise de fronteira estocástica, proposto por Battese e Coelli (1995) e Coelli, Rao e Battese (1998). Esta abordagem tem a vantagem de considerar fatores aleatórios não negligenciáveis na estimativa da eficiência técnica produtiva na agricultura (BATTESE e COELLI, 1992; BATTESE e COELLI, 1995).

O modelo utilizado no estudo usa também como referência o modelo de fronteira estocástica proposto por Aigner, Lovell e Schmidt (1977) e Meeusen e Van den Broeck (1977). Este modelo tem como objetivo divi- dir o termo de erro em dois componentes: unilateral, que capta os efeitos referentes à ineficiência da firma em relação à fronteira estocástica e simétrico, que permite variações aleatórias da fronteira entre as firmas. Além disso, permite captar possíveis efeitos de erros de medida e choques exógenos ao controle da firma, assim como quaisquer outros tipos de ruído estatístico.

O modelo estocástico pode ser expresso pela seguinte expressão matemática:

$$
Y_{i}=f\left(x_{i} ; \beta\right) e^{\left(v_{i}-u_{i}\right)}, \quad i=1,2, \ldots, n
$$

$Y_{i}$ é denotado como a produção de camarão observada na firma $i$, no ano de 2011; $x_{i}$ representa o vetor $1 x \mathrm{~K}$ de insumos ou outras variáveis explicativas associadas com a firma; $\beta$ denota o vetor $K x 1$ parâmetros desconhecidos relacionados com as variáveis independentes. A composição do termo do erro neste dá-se pelos termos $v_{i}$ e $u_{i}$, sendo esses independentes entre si. $\mathrm{O}$ primeiro termo do erro representa as variações por choques aleatórios, erros de medição etc., que são por hipótese iid $\sim N\left(0, \sigma^{2}\right)$, ou seja, independentes e identicamente distribuídos, com distribuição normal de média zero e variância $\sigma^{2}$. Já o segundo termo refere-se à ineficiência técnica, que desvia a produção da firma para baixo da fronteira de produção, podendo assumir diversos tipos de distribuição, como a half-normal (BATTESE e COELLI, 1992), normal truncada (BATTESE e COELLI, 1995), exponencial (GREENE, 2005) e gama (GREENE, 2003).

A eficiência técnica deste modelo $\left(E T_{i}\right)$ é obtida pela razão entre a produção observada e a produção 
correspondente à fronteira de produção. Tem-se a seguinte equação:

$$
E T_{i}=\frac{Y_{i}}{Y_{i}^{*}}=\frac{f\left(x_{i} ; \beta\right) e^{\left(v_{i}-u_{i}\right)}}{f\left(x_{i} ; \hat{\beta}\right) e^{v_{i}}}=e^{-u_{i}}
$$

A eficiência técnica, segundo Battese e Coelli (1992), assume o valor entre zero e um e indica a distância entre o produto observado da i-ésima firma $\left(Y_{i}^{*}\right)$ e o nível do produto que poderia ser atingindo $\left(Y_{i}\right)$, fazendo-se uso da mesma cesta de insumos, caso não houvesse ineficiência. Pode-se verificar que o valor do produto observado $\left(Y_{i}\right)$ e potencial $\left(Y_{i}^{*}\right)$ estão limitados pelos ruídos estatísticos $\left(v_{i}\right)$, desta forma, caracterizando a natureza estocástica da função de produção.

Os choques exógenos são representados por vi, sendo este simétrico $(-\infty<v<\infty)$, e assume-se que esses choques são independentes e identicamente distribuídos (iid). O termo $v_{i}$ apresenta distribuição normal $\left[v \sim N\left(0, \sigma_{v}^{2}\right)\right]$, sendo capaz de assimilar os efeitos estocásticos fora do controle da firma. O termo $u_{i}$ é um componente de eficiência unilateral $\left(v_{i} \geq 0\right)$ responsável por obter a ineficiência técnica da i-ésima firma. Os termos de erro $v_{i}$ e $u_{i}$ são independentes entre si. O termo de erro $u_{i}$ mensura a insuficiência no produto, a partir do seu valor máximo, dado pela fronteira estocástica $f\left(x_{i} ; \beta\right) e^{v_{i}}$. Este termo unilateral pode seguir a distribuição meio-normal, normal truncada, exponencial e gama (AIGNER, LOVELL e SCHMIDT, 1977).

O modelo geral da função fronteira de produção usado no presente trabalho é:

$$
Y_{j}=f\left(X_{i j} ; \beta\right)+\varepsilon_{j}
$$

em que: $Y_{j}$ é o vetor de produção de camarão em toneladas das fazendas do estado do Ceará, $X_{i j}$ é o vetor de fatores de produção, $\beta$ é o vetor de parâmetros e $\varepsilon_{j}$ é o termo de erro composto na estimativa da fronteira de produção.

Entretanto, para a realização de um estudo de fronteira estocástica é necessária a definição, a priori, de uma forma funcional. Assim, a forma escolhida para a função de produção a ser estimada é a Cobb-Douglas, por ser esta amplamente utilizada na análise da eficiência no setor agrícola e ser menos susceptível aos problemas de multicolinearidade, em relação à forma funcional Translog (ALMEIDA, 2012).

De acordo com Almeida (2012), o uso da CobbDouglas apresenta vantagens, a saber: a simplicidade na estimativa dos parâmetros, pois na forma logarítmica a função Cobb-Douglas é linear nos parâmetros, tornando o ajustamento mais fácil; os coeficientes da regressão fornecem as elasticidades de produção e podem ser comparados entre si, pois são independentes das quantidades de produto e de fatores; comparativamente com a forma funcional transcendental logarítmica (Translog), a função de produção CobbDouglas apresenta um pequeno número de parâmetros a serem estimados, resultando, portanto, em maior número de graus de liberdade para os testes estatísticos, além de ser menos susceptível aos comuns problemas de multicolinearidade, na estimativa da função de produção.

O modelo empírico escolhido é estimado com a função Cobb-Douglas, em sua forma logarítmica. A estimativa da fronteira estocástica e das eficiências técnicas permite testar as hipóteses relacionadas ao efeito dos insumos incluídos no modelo sobre a produção, assim como identificar as principais causas de ineficiência. A fronteira estocástica toma a seguinte forma:

$$
\begin{aligned}
& \ln \text { ProdC }=\beta_{0}+\beta_{1} \ln \text { NFunc }+ \\
& \beta_{2} \ln \text { Nviv }+\beta_{3} \ln \text { Aviv }+v_{i}-u_{i}
\end{aligned}
$$

em que:

ProdC: compreende o valor da produção de camarão em toneladas $(\mathrm{t})$;

N Func: número total de funcionários contratados em cada firma;

Nviv: número de viveiros em operação;

Aviv: área dos viveiros em hectares;

$v_{i}$ : distúrbios aleatórios da função de produção;

$u_{i}$ : ineficiência técnica da produção;

ln: é o logaritmo natural.

A variável $v_{i}$ representa os distúrbios aleatórios da função de produção que, por hipótese, segue uma distribuição normal com média zero e variância constante, iid $\sim N\left(0, \sigma^{2}\right)$. O termo $u_{i}$ representa a ineficiência técnica de produção, positivo e modelado neste estudo como:

$$
u_{i}=\delta z_{i}+w_{i}
$$

Sendo $z_{i}$ um vetor de variáveis microeconômicas que explicam a ineficiência técnica, e $\delta$ é um vetor de parâmetros associado a $z_{i}$, a serem estimados. A variável $z_{1}$ corresponde à densidade de estocagem $\left(\mathrm{cam} / \mathrm{m}^{2}\right)$, Dens, de cada firma. Esta variável define a quantidade de camarão por metro quadrado no 
viveiro, a qual deve ser estabelecida de modo a fazer o uso ótimo da área, visto que a densidade de estocagem, além da capacidade de suporte do viveiro pode elevar a taxa de mortalidade, o que se constitui em ineficiência. Moss e Moss (2004) afirmam que a densidade de estocagem está diretamente relacionada ao crescimento e à sobrevivência dos camarões. Além disso, a escolha da densidade de estocagem mais apropriada para o cultivo é fundamental para a viabilidade econômica das fazendas, exercendo grande influência sobre a produtividade do sistema (JACKSON e WANG, 1998).

Já a variável $z_{2}$ caracteriza a potência de aeradores por área (HP/ha), PAer. O aerador é utilizado para oxigenar o viveiro, essencial para a sobrevivência do camarão em cativeiro. Desta forma, quanto maior for a potência do aerador, maior será a quantidade de oxigênio incorporado na água. Porém, a aeração dos viveiros deve ser feita em nível adequado para não causar estresse aos camarões durante o cultivo e, assim, evitar a ineficiência produtiva (DPA/MAPA, 2001; SOUSA JÚNIOR, 2003).

As variáveis $z_{3}$ e $z_{4}$ são variáveis dummies que representam as variáveis qualitativas recebimento de assistência técnica (Atec) e uso de bandejas de alimentação (Band), respectivamente. Segundo Silva e Sampaio (2009) a colocação das rações através do sistema de bandejas reduz o desperdício e ajuda a diminuir a formação de sedimentos no fundo dos viveiros, e o elevado nível de resíduos pode provocar a mortalidade de camarões. A assistência técnica atua na prevenção e correção de manejos inadequados e má uso da tecnologia, visando reduzir problemas geradores de ineficiência.

\subsection{Testes realizados}

\subsubsection{Teste da forma funcional}

O modelo de fronteira estocástica foi estimado com as funções de produção na forma Cobb-Douglas e Translog. De acordo com teste da razão de verossimilhança (LR), a função Cobb-Douglas foi a escolhida para a análise por ajustar melhor os dados.

O teste da razão de verossimilhança (LR) tem como hipótese nula, $\mathrm{H}_{0}$ : LL Cobb-Douglas, e hipótese alternativa, $\mathrm{H}_{1}$ : LL Translog. O LR é calculado pela seguinte equação: $\mathrm{LR}=-2\left[\ln \mathrm{LL} \mathrm{H}_{0}-\ln \mathrm{LL} \mathrm{H}_{1}\right] \sim \chi^{2}$; no qual LL é o log-verossimilhança estimado para cada forma funcional. Utilizando os valores críticos mostrados por Koddee Palm (1986), falha-se em rejeitar $\mathrm{H}_{\mathrm{o}}$, se LR < T KP (Tabela de Kodde e Palm, 1986).

\subsubsection{Efeito da ineficiência técnica na função de produção}

Utiliza-se também a razão de verossimilhança (LR) para testar se as variáveis de ineficiência são responsáveis pela ineficiência técnica no modelo. Neste teste, as hipóteses são definidas como seguem: $\mathrm{H}_{0}$ : Inexistência de ineficiência técnica, $\gamma=0$; e $H_{1}$ : Existência de ineficiência técnica, $\gamma>0$. Caso a hipótese nula não seja rejeitada, o modelo de ficiência pode ser estimado pelo estimador de mínimos quadrados ordinários; caso contrário, estima-se o modelo de eficiência estocástica utilizando a função de produção selecionada.

Para realizar o teste LR, estima-se o valor da log-verossimilhança do modelo estimado sem incluir as variáveis de ineficiência e novamente aplica o teste de verossimilhança generalizada. A significância do teste consiste em comparar o LR calculado com o valor crítico da tabela de Kodde e Palm (1986), tendo o número de variáveis de ineficiência, como o grau de liberdade do teste. Decidiu-se por não rejeitar a $\mathrm{H}_{0}$, quando o LR < T KP (Tabela de Kodde e Palm, 1986).

\section{Resultados e discussão}

Nesta seção serão descritos os testes de hipóteses realizados, a estimação da fronteira de produção estocástica e os causadores de ineficiência.

\subsection{Escolha da forma funcional}

Primeiramente, para analisar os testes de hipóteses realizados, vale ressaltar que várias especificações alternativas à Cobb-Douglas foram estimadas e realizados testes com a distribuição half normal e normal truncada (BATTESE e COELLI, 1992). Contudo, como se pretendia analisar os níveis de eficiências do modelo foi necessário usar o modelo proposto por Battese e Coelli (1995), que utiliza a distribuição normal truncada.

Foi estimado o modelo Cobb-Douglas e o Translog sem progresso técnico, já que correspondia a um único tempo, realizando-se, assim, uma análise cross-section. 
Porém, após as estimações dos modelos, foi verificado que o modelo Translog não apresentou robustez, pois quase todas as variáreis independentes não foram capazes de explicar a produção; entretanto, o modelo Cobb-Douglas mostrou-se bem mais ajustado, apresentando todos os coeficientes do modelo significativos, a um nível de significância de 5\%. Para maior confiabilidade em qual forma funcional utilizar, foi realizado o teste de máxima verossimilhança e obteve-se o valor do $\lambda$, que se encontra na Tabela 2 , e a partir disso pode-se observar que o valor de $\lambda(10,915)$ é menor do que o valor crítico $(11,911)$, ao nível de $5 \%$ de significância na tabela de Kodde e Palm (1986), dado seu grau de liberdade. Desta forma, não se pode rejeitar a hipótese nula, estabelecendo que a função Cobb-Douglas apresentou-se como forma funcional mais adequada a um nível de significância de 5\%, por isso foi escolhida para representar o modelo de fronteira estocástica.

\subsection{Estimação da fronteira de produção e do modelo de ineficiência técnica}

Foi escolhida a forma funcional para a realização do teste de Inexistência de Eficiência Técnica, em que não se deve utilizar o modelo de fronteira estocástica, caso $\gamma=0$, ou seja, o modelo não é capaz de mensurar efeito de ineficiência. Foi realizado o teste de máxima verossimilhança e, a partir da estimação da fronteira, obteve-se o valor do $\lambda$, que está na Tabela 2 . Foi verificado que o valor de $\lambda(20,383)$ é maior do que o valor crítico $(11,911)$, ao nível de $5 \%$ de significância na tabela de Kodde e Palm (1986), dado seu grau de liberdade. Portanto, rejeita-se a hipótese nula que estabelece a inexistência da eficiência técnica no modelo determinado a um nível de significância de 5\%, o que justifica a estimativa do modelo de eficiência estocástica.

$\mathrm{Na}$ Tabela 3 apresentam-se os resultados das estimações do modelo, que corresponde à fronteira de produção na forma funcional Cobb-Douglas, com dis- tribuição normal truncada (BATTESE e COELLI, 1995), que foi o modelo que melhor se ajustou aos dados, após os devidos testes já especificados.

$\mathrm{Na}$ fronteira estimada foi estabelecido previamente o nível de significância de 5\% para analisar todas as estimações; porém, todos os parâmetros são altamente significativos, até mesmo a um nível de $1 \%$. Desta forma, implicou em uma função de produção bem comportada, com todos os parâmetros com os sinais esperados. Os fatores de produção, número total de funcionários contratados, número de viveiros em operação e área média dos viveiros em hectares impactam positivamente na produção de camarão das fazendas do estado do Ceará. Isto significa que cada variável explicativa incluída no modelo está positivamente associada ao nível de produção, quando as demais variáveis são mantidas constantes (ceteris paribus).

O valor do logaritmo natural da área média dos viveiros em hectares ( $\ln$ Aviv), obteve o maior coeficiente $(0,889)$ dentre os logaritmos naturais das variáveis explicativas do modelo. Por ser o coeficiente uma medida de elasticidade, em termos médios, o aumento de $1 \%$ na área média dos viveiros da fazenda está associado a um aumento de $0,889 \%$ na produção da fazenda, ceteris paribus. Isto significa que a produção e a área média dos viveiros seguem o pressuposto de que o aumento da produção é proporcionalmente menor do que o aumento no uso do fator variável.

Já os demais fatores de produção, ln NFunc e ln Nviv obtiveram coeficientes de 0,434 e 0,529, respectivamente, portanto, inferiores em magnitude ao observado pela In Aviv. Assim, assegurado que as demais variáveis permaneçam constantes, o aumento de $1 \%$ no número de funcionários da fazenda e no número de viveiros em operação, está associado ao aumento de 0,434\% e 0,529\% na produção de camarão, respectivamente. Desta forma, essas variáveis também expressam um aumento na produção equivalentemente menor do que o aumento no uso dos fatores variáveis.

Tabela 2. Testes de inexistência de eficiência técnica e forma funcional

\begin{tabular}{|c|c|c|c|c|c|}
\hline Teste de hipótese & Hipóteses & $\begin{array}{c}\text { Grau de } \\
\text { liberdade }\end{array}$ & Valor de $\lambda$ & Valor crítico & Decisão (5\%) \\
\hline \multirow{2}{*}{ Forma funcional } & $H_{0}$ : Cobb-Douglas & \multirow{2}{*}{6} & \multirow{2}{*}{10,915} & \multirow{2}{*}{11,911} & \multirow{2}{*}{ Aceita-se $H_{0}$} \\
\hline & $H_{1}:$ Translog & & & & \\
\hline \multirow{2}{*}{$\begin{array}{l}\text { Inexistência de } \\
\text { eficiência técnica }\end{array}$} & $H_{0}: \gamma=0$ & \multirow{2}{*}{6} & \multirow{2}{*}{20,383} & \multirow{2}{*}{11,911} & \multirow{2}{*}{ Rejeita-se $H_{0}$} \\
\hline & $H_{1}: \gamma>0$ & & & & \\
\hline
\end{tabular}

Fonte: Elaboração dos autores a partir dos dados da pesquisa. 
Tabela 3. Estimativas de máxima verossimilhança dos parâmetros da fronteira de produção e do modelo de ineficiência técnica das fazendas de camarão do estado do Ceará, 2011

\begin{tabular}{|c|c|c|c|c|}
\hline \multicolumn{5}{|c|}{ Fronteira Estocástica de Produção/Modelo Battese e Coelli (1995) } \\
\hline \multicolumn{5}{|c|}{ Modelo de efeitos de ineficiência/normal-truncado } \\
\hline \multicolumn{4}{|c|}{ Número de observações: 100} & Prob $>$ Chi $2=0,000$ \\
\hline \multicolumn{4}{|c|}{ Log Verossimilhança: 71,69619 } & ET Média $=0,57918$ \\
\hline Variáveis & Coeficientes & Desvio padrão & $\mathrm{Z}$ & $P>|z|$ \\
\hline$\beta_{0}$ (Const.) & 2,366 & 0,221 & 10,682 & 0,000 \\
\hline$\beta_{1}(\ln N$ Func $)$ & 0,434 & 0,100 & 4,323 & 0,000 \\
\hline$\beta_{2}(\ln N v i v)$ & 0,529 & 0,102 & 5,201 & 0,000 \\
\hline$\beta_{3}(\ln$ Aviv $)$ & 0,889 & 0,134 & 6,617 & 0,000 \\
\hline \multicolumn{5}{|c|}{ Ineficiência } \\
\hline$\delta_{0}$ (Const.) & 4,006 & 1,101 & 3,640 & 0,000 \\
\hline$\delta_{1}($ Dens $)$ & 0,848 & 0,398 & $-2,129$ & 0,033 \\
\hline$\delta_{2}(P$ Aer $)$ & 0,099 & 0,171 & 0,577 & 0,564 \\
\hline$\delta_{3}($ Atec $)$ & $-0,213$ & 0,256 & $-0,830$ & 0,406 \\
\hline$\delta_{4}($ Band $)$ & $-0,794$ & 0,703 & $-1,129$ & 0,259 \\
\hline$\sigma^{2}$ & 0,380 & 0,190 & 1,994 & 0,046 \\
\hline$\gamma$ & 0,742 & 0,170 & 4,358 & 0,000 \\
\hline$\sigma_{u}$ & 0,531 & 0,182 & 2,923 & 0,003 \\
\hline$\sigma_{v}$ & 0,313 & 0,073 & 4,301 & 0,000 \\
\hline$\sigma_{u}^{2}$ & 0,282 & 0,193 & 1,462 & 0,144 \\
\hline$\sigma_{v}^{2}$ & 0,098 & 0,046 & 2,151 & 0,032 \\
\hline
\end{tabular}

Fonte: Elaboração dos autores a partir dos dados da pesquisa.

Observados os valores dos coeficientes, também foi possível concluir que, dentre as variáveis $\ln$ NFunc e ln Nviv, o número de viveiros em operação resulta num acréscimo produtivo de $0,095 \%$ a mais do que o número total de funcionários contratados. Essa relação já era esperada, pois nas fazendas, em sua maioria, o número de funcionários é bem aquém, comparado à quantidade de viveiros e à área dos viveiros, sendo fazendas de grande extensão.

Entre as variáveis de ineficiência incluídas no modelo (Dens, PAer, Atec e Band), apenas o coeficiente da variável Dens se mostrou significativo ao nível de $5 \%$. O coeficiente da Dens apresentou sinal positivo, significando que a diminuição da densidade de estocagem está associada à diminuição da ineficiência técnica de produção de camarão. Isto pode ser um indicativo de que a densidade de estocagem dos viveiros está acima do limite, que não pode ser excedido.

A densidade média de estocagem tem sido apontada como o maior causador de ineficiência na carcinicultura. De acordo com Sousa Júnior (2003), os carcinicultores classificados como eficientes adotavam o sistema de produção semi-intensivo, que admite den- sidade de estocagem dos viveiros de 30 a $60 \mathrm{cam} / \mathrm{m}^{2}$. No presente estudo, a média da densidade de estocagem é de $33,23 \mathrm{cam} / \mathrm{m}^{2}$, variando no intervalo de $10 \mathrm{cam} / \mathrm{m}^{2}$ e $80 \mathrm{cam} / \mathrm{m}^{2}$ e, portanto, inclui valores abaixo de $30 \mathrm{cam} / \mathrm{m}^{2}$ e acima de $60 \mathrm{cam} / \mathrm{m}^{2}$, que podem conduzir à ineficiência.

Embora estatisticamente insignificante, foi possível concluir que as variáveis, potência de aeradores por área (PAer), recebimento de assistência técnica (Atec) e uso de bandejas de alimentação (Band) não são fontes causadoras de ineficiência. Isso pode se dar devido ao fato de o pacote tecnológico, apresentado pelos assistentes técnicos, já estar bastante consolidado e o uso de bandejas de alimentação já se tornou uma tecnologia bastante difundida. Já a potência dos aeradores por área pode estar relacionado ao fato de que o dimensionamento dos aeradores está corretamente especificado para atender as necessidades dos viveiros, não se constituindo em fonte de ineficiência produtiva.

Os resultados obtidos corroboraram com os alcançados por Silva e Sampaio (2009), que verificaram a insignificância no uso de bandejas de alimentação como geradora de ineficiência do modelo. Já com 
relação ao uso de aeradores, foi observado que é uma prática de gestão que influenciava diretamente na eficiência. Porém, com os resultados obtidos nesta pesquisa pode-se concluir que a potência dos aeradores distribuída nas área produtiva não influencia na eficiência da firma.

O indicador de ineficiência técnica, $\gamma\left(=\sigma_{v}^{2} / \sigma^{2}\right)$ foi estimado em 0,742 . Isto significa que $74,2 \%$ da variância total do erro composto do modelo corresponde à variância da ineficiência técnica. Este resultado é uma evidência forte da presença de ineficiência técnica no modelo estimado para a produção de camarão cultivado no estado do Ceará.

A partir da estimação da ineficiência foram calculados os escores de eficiência das fazendas da amostra. A Tabela 4 apresenta a estatística descritiva dos escores de eficiência técnica para a amostra.

A Figura 1 apresenta a distribuição de frequência dos escores de eficiência técnica das fazendas da amostra. A figura deixa evidente que a distribuição dos escores de eficiência técnica da amostra é assimétrica, assemelhando-se ligeiramente a uma distribuição normal assimétrica à esquerda. Desta forma, os escores com maior frequência estão localizados na faixa de eficiência entre 0,68 e 0,75, onde estão situadas 34 fazendas (ou $34 \%$ da amostra). De acordo com o gráfico de distribuição de frequência acumulada, observa-se que $83 \%$ das fazendas possui eficiência técnica igual ou menor que 0,75 .

A média e mediana da eficiência técnica foi de 0,579 e 0,621 , respectivamente. A amplitude dos escores de eficiência técnica é de 0,72, variando no intervalo que vai do valor mínimo de 0,180 e máximo de 0,900 . Pelo fato de a distribuição da eficiência técnica ser enviesada, a mediana mostra-se mais adequada para a medida de tendência central da distribuição.

A literatura é omissa quanto aos critérios utilizados para definir o referencial de eficiência técnica contra o qual se mede o desempenho das fazendas. Santos et al. (2009) consideraram uma abordagem ad hoc, em que o nível de eficiência foi definido em 0,90 , ou seja, o produtor com escore igual ou acima deste valor é eficiente; caso contrário, o produtor é ineficiente.

Reconhecendo que os escores são estimados de forma endógena, nesta pesquisa evita-se definir um valor como marco arbitrário de eficiência e prefere-se avaliar a eficiência de forma relativa, ou seja, comparando os grupos de fazendas mais eficientes ao grupo das menos eficientes. Desta forma, é possível avaliar quanto uma fazenda menos eficiente tem que empreender melhorias na produção para alcançar o desempenho de uma fazenda mais eficiente.

Tabela 4. Estatística descritiva dos escores de eficiência técnica das fazendas da amostra

\begin{tabular}{cccccc}
\hline Parâmetro & Mediana & Média & Desvio padrão & Mínimo & Máximo \\
\hline Eficiência técnica & 0,621 & 0,579 & 0,018 & 0,180 & 0,900 \\
\hline
\end{tabular}

Fonte: Elaboração dos autores a partir dos dados da pesquisa.

Figura 1. Distribuições de frequência absoluta e acumulada dos escores de eficiência técnica da produção da amostra

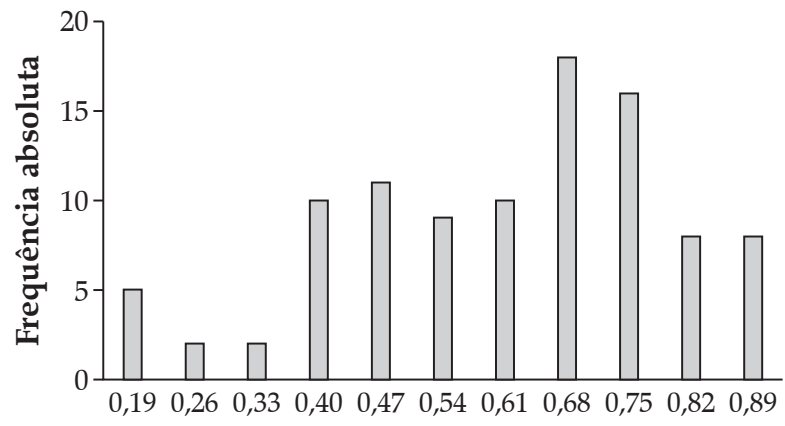

Eficiência técnica

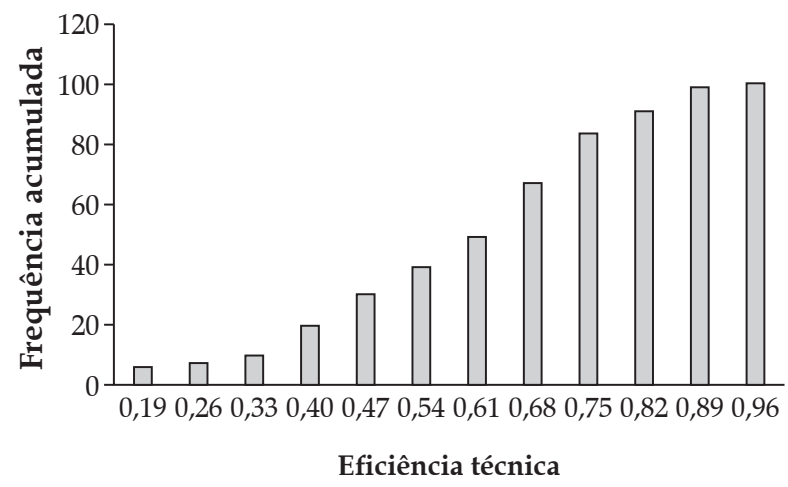

Eficiência técnica

Fonte: Elaboração dos autores a partir dos dados da pesquisa. 
Com base nos escores de eficiência técnica das fazendas, a fazenda com maior eficiência técnica obteve o escore de 0,900. Considerando-se que 99 fazendas da amostra $(N=100)$ tiveram escores inferiores a 0,900 , isto significa que $99 \%$ da amostra tem potencial de melhorar seu desempenho técnico para, assim, alcançar o nível máximo de eficiência. Desta forma, tomando a fazenda com maior escore como referencial, uma fazenda com escore mediano teria que aumentar sua eficiência técnica em $31 \%(=1-0,621 / 0,900)$ ao ponto de se tornar eficiente. De forma análoga, tomando a eficiência técnica mediana como referência, a fazenda com o menor nível de eficiência teria que aumentar sua eficiência em $71 \%$ (= $1-0,180 / 0,621)$, que pode ser considerada baixa, quando comparada com o maior escore de eficiência $(0,900)$.

Portanto, a metade da amostra (50\%) com escores igual ou superior à mediana teria que aumentar sua eficiência no máximo em $31 \%$, enquanto outra metade da amostra com escores menores que a mediana teria que aumentar sua eficiência em mais que o dobro das fazendas mais eficientes. Isto revela a desigualdade marcante da distribuição de eficiência técnica entre as fazendas da amostra.
A Tabela 5 apresenta a estatística descritiva da eficiência técnica dos quartis da amostra. A média dos escores da eficiência técnica dos quartis são $0,329\left(1^{\circ}\right), 0,534$ $\left(2^{\circ}\right), 0,665\left(3^{\circ}\right)$ e $0,788\left(4^{\circ}\right)$. O segundo e o terceiro quartis apresentam os menores desvios padrões, ou seja, estão mais concentrados em torno da média. As taxas de melhoria da eficiência técnica decrescem do 1 o para o 4 o quartil, variando no intervalo que vai de $63 \%$ a $12 \%$. As fazendas do primeiro quartil, com média de 0,329, teriam que melhorar a eficiência de produção em $63 \%$.

Na Tabela 6 tem-se uma comparação dos cinco produtores mais eficientes em relação aos cinco menos eficientes. Nesta comparação, os produtores $25,81,11,20$ e 75 serviram de benchmarks para os produtores 86, 90, 48, 27 e 38. As mesmas variáveis utilizadas na estimação do modelo de fronteira estocástica foram utilizadas como medida de comparação, ou seja, produção como variável dependente e número total de funcionários contratados em cada firma, número de viveiros em operação e área dos viveiros em hectares como variáveis explicativas.

Como pode ser observado na Tabela 6, o produtor 25, que é o mais eficiente, possui uma produção de camarão em toneladas (ProdC) maior que quase todos os produtores citados, com exceção dos produtores 81

Tabela 5. Estatística descritiva da eficiência técnica dos quartis da amostra

\begin{tabular}{|c|c|c|c|c|c|c|}
\hline Quartil & N. & Média & Desvio Padrão & Min & Max & Taxa de Melhoria \\
\hline $1^{\mathrm{O}}$ & 25 & 0,329 & 0,090 & 0,180 & 0,435 & $63 \%$ \\
\hline $2^{-}$ & 25 & 0,534 & 0,055 & 0,436 & 0,619 & $41 \%$ \\
\hline $3^{\mathrm{o}}$ & 25 & 0,665 & 0,023 & 0,624 & 0,705 & $26 \%$ \\
\hline $4^{\mathrm{o}}$ & 25 & 0,788 & 0,056 & 0,705 & 0,900 & $12 \%$ \\
\hline Total & 100 & 0,579 & 0,180 & \multicolumn{2}{|c|}{-} & $36 \%$ \\
\hline
\end{tabular}

Fonte: Elaboração dos autores a partir dos dados da pesquisa.

Tabela 6. Comparação dos cinco produtores mais eficientes com os cinco menos eficientes

\begin{tabular}{|c|c|c|c|c|c|c|c|}
\hline Ranking ${ }^{1}$ & Produtor & ET & $\begin{array}{l}\text { ProdC } \\
\text { (t) }\end{array}$ & NFunc & Nviv & $\begin{array}{l}\text { Aviv } \\
\text { (ha) }\end{array}$ & $\begin{array}{c}\text { Dens } \\
\left(\mathrm{cam} / \mathrm{m}^{2}\right)\end{array}$ \\
\hline $1^{\mathrm{O}}$ & 25 & 0,9003 & 210,00 & 8 & 5,0 & 2,0 & 55 \\
\hline $2^{-}$ & 81 & 0,8571 & 280,00 & 10 & 17,0 & 1,7 & 40 \\
\hline 30 & 11 & 0,8559 & 45,00 & 2 & 4,0 & 1,0 & 30 \\
\hline $4^{\mathrm{o}}$ & 20 & 0,8538 & 150 & 9 & 0,5 & 6 & 18 \\
\hline $5^{\mathrm{o}}$ & 75 & 0,8508 & 168 & 11 & 12 & 1,2 & 50 \\
\hline $96^{\mathrm{o}}$ & 86 & 0,1892 & 6 & 4 & 3 & 1,5 & 33 \\
\hline $97^{\circ}$ & 90 & 0,1889 & 8,4 & 5 & 2 & 2,7 & 40 \\
\hline $98^{\circ}$ & 48 & 0,1873 & 4 & 3 & 4 & 1 & 35 \\
\hline $99^{\circ}$ & 27 & 0,1838 & 30 & 6 & 5 & 4,5 & 14 \\
\hline $100^{\circ}$ & 38 & 0,1803 & 730 & 116 & 25 & 12 & 15 \\
\hline
\end{tabular}

${ }^{1} \mathrm{O}$ ranking das eficiências de todos os cem produtores se encontra com os autores, e está disponível para consulta.

Fonte: Elaborado pelos autores a partir dos dados da pesquisa. 
e 38. Entretanto, ele não faz uso de elevados quantitativos dos insumos, ou seja, comparando-se sua área média dos viveiros em operação em hectares (Aviv) com a dos produtores 20, 90 e 27 , verifica-se que o produtor 25 faz uso da Aviv (ha) de forma bem mais eficiente que os três citados anteriormente, pois este possui área média de viveiros menor e alcança produção de camarão superior.

Foi verificado que os dois produtores menos eficientes, produtores 27 e 38, têm sua eficiência fortemente influenciada pela densidade de estocagem (Dens), pois utilizavam uma densidade de estocagem abaixo do limite inferior utilizado pelos produtores eficientes estudados por Sousa Júnior (2003), que era de no mínimo $30 \mathrm{cam} / \mathrm{m}^{2}$.

Em relação ao número de funcionários contratados (NFunc) e o número de viveiros em operação (Nviv), vale ressaltar o produtor 81, que tem dois funcionários contratados e 12 viveiros em operação a mais do que o produtor 25; no entanto, possui somente $70 \mathrm{t}$ de produção de camarão a mais que este.

Pode-se também observar, com relação ao NFunc, que o produtor 20 possui maior número de funcionários contratados; porém, tem produção menor que a obtida pelo carcinicultor mais eficiente. O produtor 25 tem um NFunc menor, correspondente a menos da metade da média de funcionários contratados, que é de 16,39 .

Por fim, observando-se a variável número de viveiros em operação (Nvivi), o produtor 25 tem uma quantidade pequena, comparada à média, que é de 11,91 (Tabela 1). Mesmo assim, comparando-se à maioria daqueles que utilizam um número maior ou igual de viveiros, como os produtores 27 e 75, o produtor 25 tem produção bem maior, chegando a ser sete vezes maior que a obtida pelo produtor 27.

Com base nessas informações, pode-se afirmar que as causas para o uso ineficiente dos insumos estão relacionadas diretamente com as três variáveis explicativas utilizadas no modelo NFunc, Nviv e Aviv, pois o uso excessivo destas tem reduzido a eficiência das firmas. Contudo, comparando o produtor mais eficiente com o menos eficiente, verifica-se que a variável que causa maior impacto na eficiência é NFunc, observando, assim, que o uso excessivo de mão de obra pode ser a principal causa de a eficiência das fazendas de carcinicultura do estado do Ceará estar se reduzindo.

\section{Considerações finais}

Com base no modelo de fronteira de produção estocástica para a carcinicultura cearense, as elasticidades da produção determinada pela mudança nas quantidades dos insumos investigadas foram positivas; porém, menores que a unidade. A maior elasticidade da produção foi observada para a variação percentual na área média dos viveiros, ceteris paribus, relativamente aos outros insumos investigados.

Dentre as variáveis que foram incluídas para explicar a ineficiência técnica, a densidade média de estocagem foi a única variável que se mostrou significativa. $\mathrm{O}$ aumento da quantidade de camarão estocado por metro quadrado está associado positivamente à ineficiência da produção de camarão.

A distribuição da eficiência técnica da produção de camarão cultivado no Ceará mostrou-se assimétrica e negativamente enviesada, com $83 \%$ das fazendas amostradas experimentando nível de eficiência igual ou inferior a 0,75 . Por esta razão, a média e a mediana da eficiência foram de 0,579 e 0,621 , respectivamente. Isto significa que existe potencial de melhoria de eficiência a ser alcançada pelas fazendas e que vai depender das variáveis estudadas como determinantes da ineficiência.

Em termos médios, o grau de ineficiência dos carcinicultores pode ser considerado alto, já que uma melhoria na eficiência pode ser empreendida na fazenda no intervalo que vai de $12 \%$ a $63 \%$. Isto significa que o potencial produtivo deste setor está sendo subutilizado e a tecnologia pode estar sendo mal empregada ou não se encontra ao alcance de todos os carcinicultores cearenses. Tal situação compromete $o$ crescimento da produção e reduz a competitividade do setor nos mercados nacional e internacional.

Para pesquisas futuras, sugere-se ampliar o número de variáveis no modelo, explicativas e fontes de ineficiência, abrangendo aspectos da produção, gerenciamento e gestão ambiental. Essas variáveis adicionais permitirão ampliar a compreensão das relações envolvidas na função de produção do camarão cultivado.

\section{Referências}

ABCC - Associação Brasileira de Criadores de Camarão. Censo setorial do camarão em 2011. Disponível 
em: <http://www.panoramadaaquicultura.com.br/ novosite/?p=1311>. Acesso em: 3 out. 2015 .

Levantamento da infraestrutura produtiva e dos aspectos tecnológicos, econômicos, sociais e ambientais da carcinicultura marinha no Brasil em 2011, 2013. Disponível em: <http://abccam.com.br/site/ wpcontent/uploads/2013/12/LEVANTAMENTO-DAINFRAESTRUTURA-PRODUTIVA.pdf> . Acesso em: 03 out. 2015.

AIGNER, D. J., LOVELL, C. A. K. e SCHMIDT, P. Formulation and estimation of stochastic frontier production function models. Journal of Econometrics, v. 6, p. $21-37,1977$.

ALMEIDA, P. N. A. Fronteira de produção e eficiência técnica da agropecuária brasileira em 2006. Tese (Doutorado) - Curso de Economia Aplicada, Escola Superior de Agricultura "Luiz de Queiroz", Universidade de São Paulo, Piracicaba, 2012.

ARAÚJO, D. C. Avaliação do programa nacional de desenvolvimento da aquicultura: o caso da Carcinicultura Marinha no Nordeste. Dissertação (Mestrado em Gestão Pública para o Desenvolvimento do Nordeste), UFRPE/SUDENE/PNUD, Recife, 2001.

ARAÚJO, A. M. M. e ARAÚJO, R. C. P. Análise das práticas de gestão ambiental e seus impactos sobre a produtividade da carcinicultura no Ceará. Anais... Congresso da sociedade Brasileira de Economia, Administração e Sociologia Rural - SOBER, 53, Paraíba, João Pessoa, jul. 2015, p. 19.

BATTESE, G. E. e COELLI, T. J. Frontier production functions, technical efficiency and panel data: with application to paddy farmers in India. Journal of Productivity Analysis, n. 3, p. 153-169, 1992.

BATTESE, G. E. e COELLI, T. J. A model for technical inefficiency effects in a stochastic frontier production function for panel data. Empirical Economics, v. 20, issue 2, p. 325-332, 1995.

BEGUM, M. E. A., HOSSAIN, M. I. e PAPNAGIOTO, E. Technical efficiency of shrimp farming in Bangladesh: an application of the stochastic production frontier approach. Journal of the World Aquaculture Society, v. 44, n. 5, p. 641-654, 2013.

CEARÁ. Instituto de Pesquisa e Estatística Econômica do Ceará - IPECE. (Org.). PIB trimestral do Ceará, 2014. Disponível em: <http://www.ipece.ce.gov.br/ categoria2/pib/APRESENTACAO_PIB_2o_TRIM2014. pdf>. Acesso em: 27 out. 2014.

COELLI, T. J., RAO, P., D. S. e BATTESE, G. E. An introduction to efficiency and productivity analysis. Boston: Kluwer Academic Publishers, 1998.
CONCEIÇÃO, P. H. Z. e CONCEIÇÃO, J. C. P. R. Uma contribuição metodológica da produtividade total dos fatores na agricultura brasileira, 2005. SOBER. Disponível em: <www.sober.org.br/palestras > Acesso em: 07 jun. 2016.

DEY, M. M. et al. The impact of integrated aquacultureagriculture on small-scale farms in Southern Malawi. Agricultural Economics, Malden, v. 41, n. 1, p. 67-79, 2010.

DPA/MAPA - Departamento de Pesca e Aquicultura do Ministério da Agricultura Pecuária e do Abastecimento e ABCC - Associação Brasileira de Criadores de Camarão. Plataforma tecnológica do camarão marinho cultivado. Brasília-DF, out. 2001. 276p.

FAO - Food and Agiculture Organization of United Nations. The state of world fisheries and aquaculture. Roma SOFIA, p. 24-26, 2012.

. Seção fisheries and aquaculture department, Roma SOFIA, 2014.

FARREL, M. J. The measurement of production efficiency. Journal of the Royal Statistical Society, Series A (General), v. 120, n. 3, 1957.

FONSECA, G. B. N. G. Gestão da qualidade no arranjo produtivo da carcinicultura no estado do Rio Grande do Norte. Tese (Doutorado em Ciência Animal) - Universidade Federal de Minas Gerais, Escola de Veterinária. Belo Horizonte, 2005. 96 p.

GREENE, W. H. Econometric Analysis. 3. ed. New Jersey: Prentice Hall, 2003. 914 p.

GREENE, T. G. Bridging the great divide: exploring the Relationship between Student Engagement and Educational Outcomes for African American and Hispanic Community College Students in the State of Florida. Dissertação, The University of Texas at Austin, Florida, dec. 2005.

ISLAM, G. M. N., YEW, T. S. e NOH, K. M. Technical efficiency analysis of shrimp farming in Peninsular Malaysia: a stochastic frontier production function approach. Trends in Applied Sciences Research, v. 9, n. 2, p. 103, 2014.

JACKSON, C. J. e WANG, Y. G. Modelling growth rate of Penaeus monodon Fabricius in intensively managed ponds: effects of temperature, pond age and stocking density. Aquacult. Res., n. 29, p. 27-36, 1998.

JONES, C. M. e DOURADO, J. D. A. Aumento da produtividade da carcinicultura e redução de lançamentos de resíduos. Anais... Congresso sobre Planejamento e Gestão da Zona Costeira dos Países de Expressão Portuguesa, 2, 2003. 
KODDE, D. A. e PALM, F. C. Wald criteria for jointly testing equality and inequality restrictions. Econometric, Notes and Comments, v. 54, n. 5, p. 1243-1248, 1986.

LIU, C., LAPORTE, A. e FERGUSON, B. S. The quantile regression approach to efficiency measurement: insights from Monte Carlo simulations. Health Economics, York, v. 17, n. 9, p. 1073-1087, set. 2008.

LOVELL, C. A. K. e SCHIMIDT, P. A. Comparasion of alternative approaches to the measuremente of productive efficiency. In: Applications of modern production theory. Boston: Kluwer Academic Publishers, p. 3-32, 1988.

MAGALHÃES, M. S. E. Cultivo de Litopenaeus vannamei (BOONE, 1931) em sistema multifásico. Dissertação (Mestrado em Recursos Pesqueiros e Aquicultura) Universidade Federal Rural de Pernambuco, Recife, 2004. $60 \mathrm{p}$.

MEEUSEN, W. e BROECK, J. V. D. Efficiency Estimation from Cobb-Douglas Production Functions with Composed Error. International Economic Review, n. 18, p. 435-444, 1977.

MOSS, K. K. e MOSS. S. M. Effects of artificial substrate and stocking density on the nursery production of pacific white shrimp, Litopenaeus vannamei. Journal of the World Aquaculture Society, n. 35, p. 536-542, 2004.

OLIVEIRA, I. R. Utilização da análise envoltória de dados (DEA), no diagnóstico da eficiência de cultivo do camarão marinho Litopenaeus vannamei (BOONE, 1931). Dissertação (Mestrado em Biometria e Estatística Aplicada) - Universidade Federal de Pernambuco, Recife, 2008. $51 \mathrm{p}$.

OSTRENSKY, A. e PESTANA, D. Avaliação das taxas de crescimento de Farfantepenaeus paulensis (PérezFarfante, 1967) em viveiros de cultivo. Archives of Veterinary Science, v. 5, n. 1, p. 5-15, 2000.

RASHID, M. H. A. e CHEN, J. Technical efficiency of shrimp farmers in Bangladesh: a stochastic frontier production Function analysis. Bangladesh Journal of Agricultural Economics XXV, n. 2, p. 15-31, 2002.

REINHARD, S., LOVELL, C. A. K. e THIJSSEN, G. J. Environmental efficiency with multiple environmentally detrimental variables estimated with SFA and DEA. European Journal of Operational Research, v. 121, 2000, p. 287-303.

REIS, R. P., RICHETTI, A. e LIMA, A. L. R. Eficiência econômica na cultura do café: um estudo no sul de Minas Gerais. Organizações Rurais \& Agroindustriais, Lavras, v. 7, n. 1, p. 50-59, 10 set. 2005.

RIVERA, E. B. B. de R. e COSTANTIN, P. D. Produtividade total dos fatores nas principais lavouras de grãos brasileiras: análise de fronteira estocástica e índice de Malmquist. São Paulo-SP, 2007. 18 p. Centro de Ciências Sociais e Aplicadas, Universidade Presbiteriana Mackenzie, 2007. ROCHA, I. P. e RODRIGUES, J. A carcinicultura brasileira em 2002. Revista da Associação Brasileira de Criadores de Camarão, ano 5, n. 1, p. 30-45, 2003.

RODRIGUES, J. e BORBA, M. Carcinicultura brasileira: estatísticas e revelações. Revista Feed $\mathcal{E}$ Food, ano VII, n. 72, abr. 2013. Disponível em: < http:// www.revistafeedfood.com.br/pub/curuca/index. jsp?ipg=86345>. Acesso em: 04 out. 2015.

SAMPAIO, Y. e COUTO, É. Geração de empregos diretos $e$ indiretos na cadeia produtiva do camarão marinho cultivado. Universidade Federal de Pernambuco, Recife, fev. 2008. Disponível em: <http://abccam.com. br/site/wp-content/uploads/2012/09/GERAO_DE_ EMPREGOS_-_tese.pdf $>$. Acesso em: 07 jun. 2016.

SANTOS, V. F. et al. Análise da eficiência técnica de talhões de café irrigados e não-irrigados em Minas Gerais: 2004-2006. Revista de Economia e Sociologia Rural, v. 47, n. 3, 2009, p. 677-698.

SANTOS, C. J. A., SANTOS, D. L. e MENDES, P. P. Uso de modelos matemáticos para avaliação das variáveis de manejo de Litopenaeus vannamei (BOONE, 1931). ACTAPESCA-Acta fisheries and aquaculture/Acta Pesca $e$ Aquicultura, v. 2, n. 2, 2015.

SILVA, F. D. V., CARVALHO, R. M. e CAMPOS, R. T. Análise da eficiência técnica dos produtores de leite do estado do Ceará. Anais... Congresso da SOBER, 55, Londrina, 2007. 21p.

SILVA, J. L. M. e SAMPAIO, L. M. B. Eficiência, gestão e meio ambiente na carcinicultura do Rio Grande do Norte. Revista de Economia e Sociologia Rural, v. 47, n. 4, p. 883-902, 2009.

SIVARAMAN, I. et al. Technical efficiency of shrimp farming in Andhra Pradesh: estimation and implications. Current World Environment, v. 10, n. 1, p. 199-205, 2015.

SOUZA JUNIOR, J. P. Análise da eficiência da produção de camarão marinho em cativeiro no estado do Ceará. Dissertação (Mestrado em Economia Rural) Universidade Federal do Ceará. Fortaleza, Ceará, 2003.

TANNURI-PIANTO, M. E., SOUSA, M. C. S. e ARCOVERDE, F. D. Fronteiras de eficiência estocástica para as empresas de distribuição de energia elétrica no Brasil: uma análise de dados de painel. Estudos Econômicos, v. 39, n. 1, p. 221-247, 2009.

TUPY, O. e YAMAGUCHO, L. C. T. Eficiência e produtividade: conceitos e medição. Revista Agricultura em São Paulo, São Paulo-SP, v. 45, p. 39-51, 1998.

Todo o conteúdo deste periódico, exceto onde estiver identificado, está licenciado sob uma Licença Creative Commons (cc by 4.0 )

RESR, Piracicaba-SP, Vol. 56, № 01, p. 035-050, Jan./Mar. 2018 - Impressa em Abril de 2018 